\title{
Incorporation of School Absenteeism Data into the Maryland Electronic Surveillance System for the Early Notification of Community-based Epidemics (ESSENCE)
}

\author{
Zachary Faigen*, Anikah Salim and Isaac Ajit \\ Office of Preparedness \& Response, Maryland Department of Health and Mental Hygiene, Baltimore, MD, USA
}

\section{Objective}

The state of Maryland has incorporated $100 \%$ of its public school systems into a statewide disease surveillance system. This session will discuss the process, challenges, and best practices for expanding the ESSENCE system to include school absenteeism data as part of disease surveillance. It will also discuss the plans that Maryland has for using this new data source, as well as the potential for further expansion.

\section{Introduction}

Syndromic surveillance offers the potential for earlier detection of bioterrorism, outbreaks, and other public health emergencies than traditional disease surveillance. The Maryland Department of Health and Mental Hygiene (DHMH) Office of Preparedness and Response $(\mathrm{OP} \& \mathrm{R})$ conducts syndromic surveillance using the Electronic Surveillance System for the Early Notification of Community-based Epidemics (ESSENCE). Since its inception, ESSENCE has been a vital tool for DHMH, providing continuous situational awareness for public health policy decision makers. It has been established in the public health community that syndromic surveillance data, including school absenteeism data, has efficacy in monitoring disease, and specifically, influenza activity. Schools have the potential to play a major role in the spread of disease during an epidemic. Therefore, having school absenteeism data in ESSENCE would provide the opportunity to monitor schools throughout the school year and take appropriate actions to mitigate infections and the spread of disease.

\section{Methods}

DHMH partnered with the Maryland State Department of Education (MSDE), local health departments, and local school systems to incorporate school absenteeism data into the syndromic surveillance program. There are 24 local public school systems and 24 local health departments in the state of Maryland. OP\&R contacted each local school superintendent and each local health officer to arrange a joint meeting to discuss the expansion of the ESSENCE program to include school absenteeism data. Once the meetings were arranged, OP\&R epidemiologists traveled to each local jurisdiction and presented their plan for the ESSENCE expansion. At each meeting were representatives from the local health department, as well as school health, school attendance, and school IT staff. This allowed all questions and concerns to be addressed from both sides. In addition to the targeted meetings and presentations, the Secretary of Health issued an executive order which required all local school systems to sign a memorandum of understanding (MOU) with DHMH. This MOU detailed the data elements to be shared with the ESSENCE program and the process by which this would be shared. While this order made data contribution mandatory, the site visits by the OP\&R staff created a working relationship and partnership with the local jurisdictions. Data was collected from all public schools in the state including elementary, middle, and high schools.

\section{Results}

As of June 30, 2012, Maryland became the first state in the United States to incorporate $100 \%$ of its public school systems $(1,424$ schools) into ESSENCE. Each school system reports absenteeism data daily via an automated secure FTP (sFTP) transfer to DHMH. Due to its unique properties, Johns Hopkins Applied Physics Laboratory (JHUAPL) designed a new detection algorithm in ESSENCE specifically for this data source. OP\&R epidemiologist review and analyze this data for disease surveillance purposes in conjunction with other data sources in ESSENCE (emergency department chief complaints, poison control center data, thermometer sales data, and over-the-counter medication sales data). Integrating school absenteeism data will provide a more complete analysis of potential public health threats. The process by which Maryland incorporated their public school systems' data could potentially be used as a best practice for other jurisdictions. Not only was DHMH able to obtain data from all public schools in the state, but the process also enhanced collaboration between local health departments and public school systems.

\section{Keywords \\ ESSENCE; Surveillance; Absenteeism}

\section{Acknowledgments}

The Office of Preparedness \& Response at the Maryland Department of Health and Mental Hygiene would like to acknowledge and thank the Maryland State Department of Education and the 24 local health departments and public school systems for their support and collaboration to successfully implement this project.

\section{*Zachary Faigen}

E-mail: zfaigen@dhmh.state.md.us 